NUREG/CR-0064

LA-7306-MS

Informal Report

R4

\title{
One-Dimensional Calculation of Flow Branching Using the Method of Characteristics
}

\author{
R. W. Meier \\ R. G. Gido
}

Manuscript submitted: May 1978

Date published: May 1978

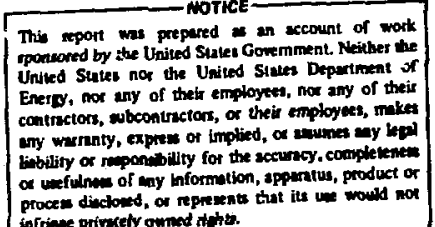

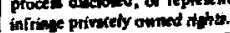

Prepared for

Division of Systems Safety

Office of Nuciear Resctor Regulation

US Nuclear Regulatory Commission

Washington, DC 20555 


\title{
ONE-DIMENSIONAL CALCULATION OF FLOW BRANCHING \\ USING THE METHOD OF CHARACTERISTICS
}

by

R. W. Heier

R. G. Gido

\begin{abstract}
In one-dimensional flow systems, the flow often branches, such as at a tee or manifold. This study develops a formulation for calculating the flow through branch points with one-dimensional method of characteristics equations. The resultant equations were verified by comparison with experimental measurements.
\end{abstract}

\section{INTRODUCTION}

The purpose of this study is to develop a subroutine that can be added to the COMPARE-Mod 2 code for modeling transient flow through branching points in flow systems. COMPARE-Mod 2, currently under development at LASL, will feature the capability of solving transient compressible duct flow problems with the method of characteristics. ${ }^{1}$ The code will include the basic features of the COMPARE $^{2}$ and COMPARE-Mod $7^{3}$ codes.

The procedures developed are based on the method of characteristics that is used to solve the flow in each of the legs or vents in the COMPARE-Mod 2 code. As formulated, all of the legs are assumed to have their first axial location node at the branch point. The branch point, $P$, is shown schematically in Fig. 1 for a system with three legs and a flow configuration in which the flow in Junction (vent) 1 is toward the branch and the flows in Junctions 2 and 3 are away from the branch point. The junctions are separated by a central volume of undefined extent, that is, a branching point with no volume.

The flow patterns established at branch points are extremely complex, and it is impossible to develop accurate one-dimensional theories to model the flow. 


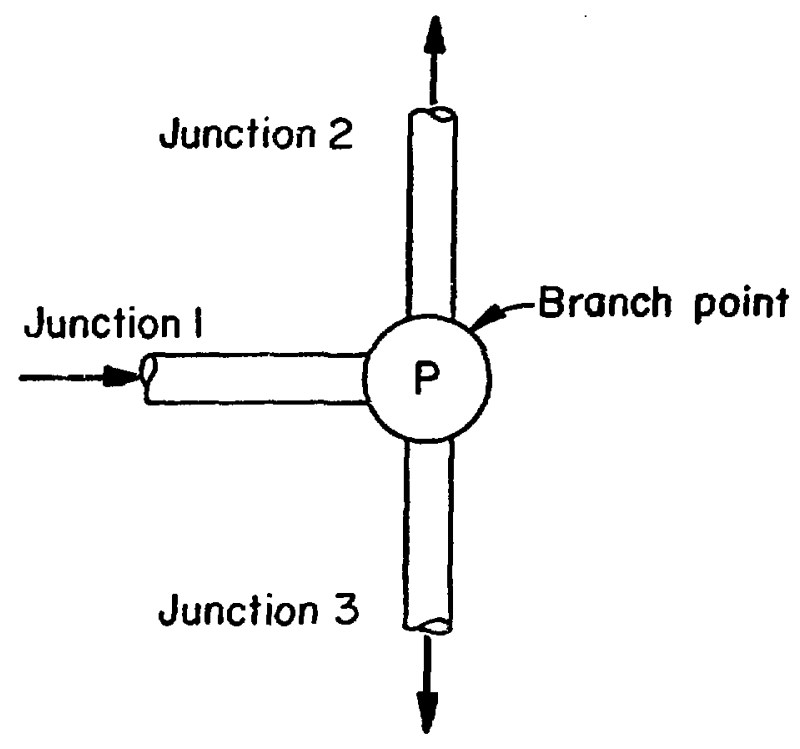

Fig. 1. Branching flow with three junctio,s.

However, this study shows that it is possible to account for branching flow systems within the limitations of the one-dimensional analysis of the COMPARE code by proper selection of head loss multipliers.

II. DERIVATION OF CHARACTERISTIC EQUATIONS FOR BRANCHING FLOW The one-dimensional unsteady continuity equation for compressible flow is 4

$$
\frac{\partial \rho}{\partial t}+\frac{\partial u}{\partial x}+u \frac{\partial \rho}{\partial x}=0
$$

and the equation for conservation of momentum is

$$
\frac{\partial u}{\partial t}+u \frac{\partial u}{\partial x}+\frac{1}{\rho} \frac{\partial p}{\partial x}+F=0
$$

The effects of friction and buoyancy are included in the F term,

$$
F=\frac{f u|u|}{2 D}+\cos \alpha
$$

The conservation of energy equation can be written

$$
\frac{\partial h}{\partial t}+u \frac{\partial h}{\partial x}=Q+u F+\frac{1}{\rho}\left(\frac{\partial p}{\partial t}+u \cdot \frac{\partial p}{\partial x}\right) .
$$


The equation of state can be written

$h=h(p, p)$,

from which

$$
\begin{aligned}
& \frac{\partial h}{\partial t}=\left(\frac{\partial h}{\partial p}\right)_{\rho} \frac{\partial p}{\partial t}+\left(\frac{\partial h}{\partial \rho}\right)_{p} \frac{\partial \rho}{\partial t} \text {, and } \\
& \frac{\partial h}{\partial x}=\left(\frac{\partial h}{\partial p}\right)_{\rho} \frac{\partial p}{\partial x}+\left(\frac{\partial h}{\partial \rho}\right)_{p} \frac{\partial \rho}{\partial x} .
\end{aligned}
$$

Substituting Eqs. (5) and (6) into Eq. (4), after some manipulation one obtains

$$
\frac{\partial p}{\partial t}+u \frac{\partial p}{\partial x}-c^{2}\left(\frac{\partial \rho}{\partial t}+u \frac{\partial \rho}{\partial x}\right)-Q^{\prime}=0
$$

where $c^{2} \equiv \frac{\left(\frac{\partial h}{\partial \rho}\right)_{p}}{\left(\frac{\partial h}{\partial p}\right)_{\rho}-\frac{T}{\rho}}$ and

$$
Q^{\prime} \equiv \frac{Q}{\left(\frac{\partial h}{\partial p}\right)_{\rho} \frac{u F}{-\frac{1}{\rho}}}
$$

Multiplying Eq. (1) by the constant $\lambda$, Eq. ( 7 ) by the constant $\gamma$, and adding them to Eq. (2) yields

$$
\begin{aligned}
& \frac{\partial u}{\partial t}+\frac{\partial u}{\partial x}(\rho \lambda+u)+\lambda\left[\frac{\partial p}{\partial t}+\frac{\partial p}{\partial x}\left(\frac{1}{\gamma \rho}+\dot{i}\right)\right] \\
& +\frac{\partial \rho}{\partial t}\left(\lambda-\gamma c^{2}\right)+\frac{\partial \rho}{\partial x} u\left(\lambda-\gamma c^{2}\right)+F-\gamma Q^{\prime}=0 .
\end{aligned}
$$


Equation (8) becomes an ordinary differential equation if

$$
\begin{aligned}
& \lambda \rho+u=\frac{d x}{d t}, \\
& \frac{1}{\gamma \rho}+u=\frac{d x}{d t},
\end{aligned}
$$

and

$$
\lambda-\gamma c^{2}=0
$$

Substitution of Eqs. (8) through (11) into Eq. (8) gives the ordinary differential equation

$$
\frac{d u}{d t}+\gamma \frac{d p}{d t}+F-\gamma Q^{\prime}=0
$$

after use of the chain rule for differentiation,

$$
\frac{d u}{d t}=\frac{\partial u}{\partial t}+\frac{\partial u}{\partial x} \frac{d x}{d t}
$$

From Eqs. (9) through (11), it can be seen that

$$
\begin{aligned}
& \lambda= \pm \frac{c}{\rho}, \\
& \gamma= \pm \frac{1}{c \rho}, \text { and } \\
& \frac{d x}{d t}=u \pm c .
\end{aligned}
$$

When $\lambda=\frac{c}{\rho}$, the so-called Family I or left-side characteristic equation ${ }^{4}$ is obtained. Equation (14) becomes

$$
\frac{d x}{d t}=u+c ;
$$

and from Eq. (8),

$$
d u+\frac{1}{c \rho} d p+\left(F-\frac{Q^{\prime}}{C \rho}\right) d t=0
$$


When $\lambda=\frac{-c}{\rho}$, the Family II or right-side characteristic equation is obtained.

$$
\begin{aligned}
& \frac{d x}{d t}=u-c . \\
& d u-\frac{1}{c \rho} d p+\left(F+\frac{Q^{\prime}}{c \rho}\right) d t=0 .
\end{aligned}
$$

When $\frac{d x}{d t}=u$, Eq. (4) becomes

$$
d h=\frac{1}{\rho} d p+(Q+u F) d t .
$$

\section{FINITE DIFFERENCE FORMULATION}

The differential equations, Egs. (15) through (19), are noniinear, because their coefficients are functions of the dependent variables, $x$ and $t$. Over a small time increment, however, the coefficients can be considered constant in a finite difference approximation. In the following finite difference formulation, the coefficients are based on the values obtained at the previous time increment. A. Momentum Equation

When the boundary is iecated at the first node of a junction as shown in Fig. 2, only the Family II characteristics apply. Therefore, along the characteristic curve P-S, which is approximated by a straight line, Eq. (17) becomes

$$
x_{S}-x_{A}=-(u-c)_{A} \Delta t
$$

where the values of $u$ and $c$ at point $A$ are used. The value of an arbitrary parameter, $Y$, at point $S$ and time $t-\Delta t$ can be obtained from

$$
\frac{y_{S}-y_{A}}{y_{B}-y_{A}}=\frac{x_{S}-x_{A}}{x_{B}-x_{A}} \equiv Z_{S} .
$$

Substituting Eq. (20) into Eq. (21),

$$
z_{s}=-\frac{(u-c)_{A} \Delta t}{x_{B}-x_{A}} \text {, }
$$




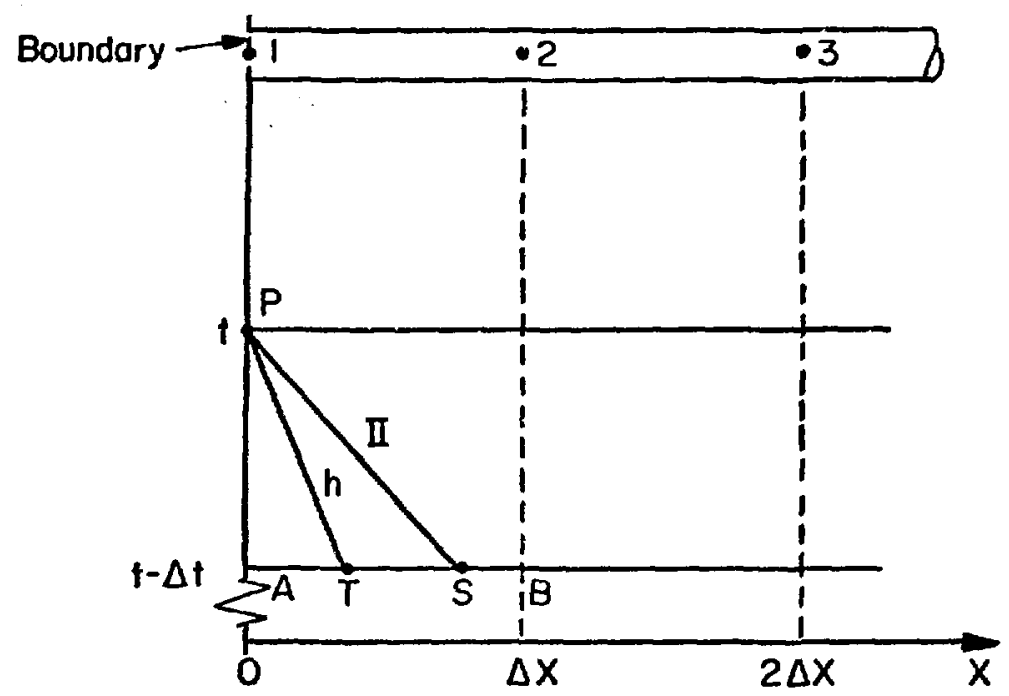

Fig. 2. Characteristic curves at a junction boundary.

and

$$
Y_{S}=Y_{A}+Z_{S}\left(Y_{B}-Y_{A}\right)
$$

Assuming that there is no heat addition to the flow, and neglecting friction and gravity forces, the characteristic Eq. (18) simplifies to

$$
d u-\frac{1}{c p} d p=0 \text {. }
$$

In finite difference form, Eq. (24) becomes

$$
u_{p}-u_{s}-\frac{1}{c_{A} \rho_{A}}\left(p_{p}-p_{S}\right)=0 \text {. }
$$

Consider a flow branch point consisting of three junctions as shown in Fig. 3. Each of the junctions has its first nodal point at the boundary of the branch and is denoted by $P_{i}$. The branch is assumed to be at a pressure $P$. The following development is presented for three junctions, but can easily be extended to an arbiträy number of junctions. 


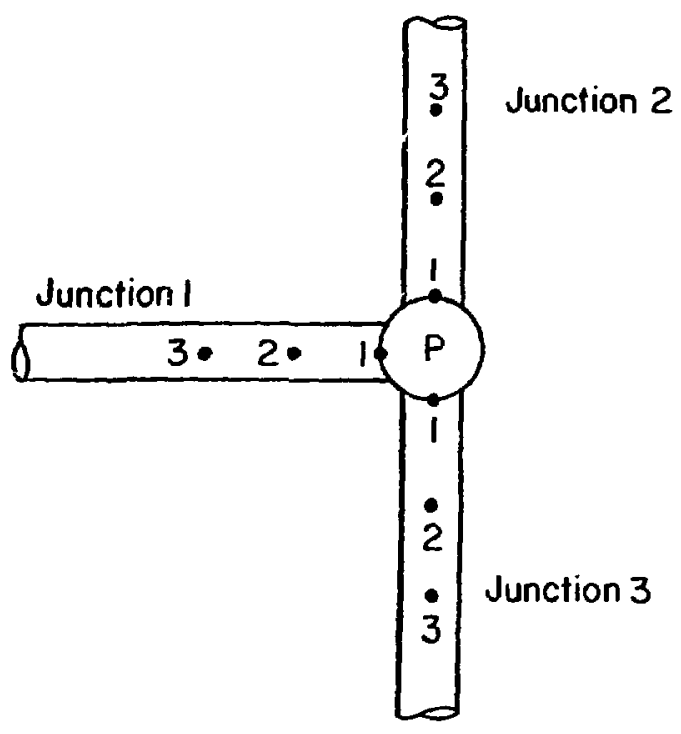

Fig. 3. Branch point with three junctions.

The characteristic equation for the branch point at the boundary of each junction can be written from Eq. (25).

$$
p_{P_{i}}=p_{S_{i}}+c_{A_{i}} p_{A_{i}}\left(u_{p_{i}}-u_{S_{i}}\right) .
$$

Defining a loss coefficient between each branch point and the branch central pressure,

$$
K_{i} \equiv \frac{P-p_{p_{i}}}{\frac{1}{2} \rho_{P_{i}} u_{p_{i}}\left|u_{p_{i}}\right|} \simeq \frac{P-p_{p_{i}}}{\frac{1}{2} \rho_{A_{i}} u_{A_{i}}\left|u_{A_{i}}\right|}
$$

Rearranging Eq. (27),

$$
p_{P_{i}}=P-\frac{\eta}{2} K_{i} \rho_{A_{i}} u_{A_{i}}\left|u_{A_{i}}\right| .
$$


Substituting Eq. (26) into Eq. (28) and rearrarging, one obtains

$$
u_{u_{i}}=\frac{P-\gamma_{i}}{\beta_{i}},
$$

where

$$
\begin{aligned}
& B_{i}=c_{A_{i}} \rho_{A_{i}} \text {, and } \\
& \gamma_{i}=p_{S_{i}}-c_{A_{i}} \rho_{A_{i}} u_{A_{i}}+\frac{1}{2} k_{i} \rho_{A_{i}} u_{A_{i}}\left|u_{A_{i}}\right| .
\end{aligned}
$$

Equation (29) represents three equations in four unknowns. A fourth equation is provided by the continuity equation for the branch. Neglecting storage within the branch,

$$
\sum_{i=1}^{3} A_{i} \rho_{A_{i}} u_{A_{i}}=0 .
$$

Substituting Eq. (29) into Eq. (32) and solving for $P$,

$$
P=\frac{\sum_{i=1}^{3} \frac{A_{\mathbf{i}} \rho_{A_{i}} \gamma_{\mathbf{i}}}{B_{\mathbf{i}}}}{\sum_{i=1} \frac{A_{\mathbf{i}} \rho_{A_{i}}}{\beta_{\mathbf{i}}}}
$$

Equation (33), along with Eqs. (30) and (31), can be solved for P, after which Eq. (29) can be solved for the branch point velocities. Equation (26) or (28) can then be used to solve for the branch point pressures. 
An alternate formulation for the reprosentation of the losses ncountered at the branch point was developed fcr compressible flow, but was not used in the verification calculations. In polytropic flow, the static pressures at two stations are related by

$$
\frac{p_{1}}{p_{2}}=\left[\frac{1-\left(\frac{k-1}{2}\right) \frac{u_{1}{ }^{2}}{c_{1}{ }^{2}+\left(\frac{k-1}{2}\right) u_{1}^{2}}}{1-\left(\frac{k-1}{2}\right) \frac{u_{2}{ }^{2}}{c_{2}{ }^{2}+\left(\frac{k-1}{2}\right) u_{2}{ }^{2}}}\right]^{\frac{a}{a-1}},
$$

where $a$ is the polytropic exponent determined from the isentropic exponent and loss coefficients. 2,3

Letting $p_{1}$ be the branch point pressure and $p_{2}$ the branch central pressure $(P)$, and assuming that the flow stagnates in the branch (a limiting assumption), Eq. (34) can be rewritten for each junction.

$$
\frac{p_{P_{i}}}{p}=\left[1-\left(\frac{k-1}{2}\right) \frac{u_{P_{i}}^{2}}{c_{p_{i}}^{2}+\left(\frac{k-1}{2}\right) u_{P_{i}}^{2}}\right]^{\frac{a_{i}}{a_{i}-1}},
$$

where

$$
a_{i}=\frac{\frac{k}{k-1}\left(1-k_{E_{i}}\right)}{\frac{k}{k-1}\left(1-k_{E_{i}}\right)-1}=\frac{k\left(1-k_{E}\right)}{1-k K_{E}}
$$

for flow from a junction to a branch point $(i=1)$, and 


$$
a_{i}=\frac{K_{I_{i}}+1}{K_{I_{i}}+\frac{1}{k}}
$$

for flow from a branch point to a junction $(i=2,3)$.

Equation (35) can then be used with Eqs. (26) and (32) to arrive at a relation similar to Eq. (33) where the coefficients are now given by

$$
\begin{aligned}
& \varepsilon_{i}=\frac{{ }_{A_{A_{j}}}{ }^{\rho} A_{i}}{\alpha_{i}}, \\
& \gamma_{i}=\frac{{ }_{P_{S_{i}}}-C_{A_{j}}{ }^{\rho} A_{i}{ }^{u} S_{i}}{\alpha_{i}},
\end{aligned}
$$

where

$$
\alpha_{i}=\left[1-\left(\frac{k-1}{2}\right) \frac{u_{A_{i}}^{2}}{c_{A_{i}}{ }^{2}+\left(\frac{k-1}{2}\right) u_{A_{i}}{ }^{2}}\right]^{\frac{a_{j}}{a_{i}-1}}
$$

The assumption of flow stagnation in Eq. (35) can be relieved by calculation of a mass-averaged velocity at the branch point and use of this velocity in the denominator of Eq. (34).

\section{B. Energy Equation}

The path of the characteristic line for the energy equation is depicted in Fig. 2 for the case where the direction of flow is from the vent interior toward the boundary at $x=0$. The characteristics curve is given by Eq. (19) and, when approximated by a straight line over the time increment $\Delta t$, can be written as

$$
x_{T}-x_{A}=-u_{A} \Delta t .
$$


Linearly, interpolating, for $u_{A}<0$,

$$
\frac{h_{T}-n_{A}}{h_{B}-h_{A}}=\frac{x_{T}-x_{A}}{x_{B}-x_{A}} .
$$

Substituting Eq. (36) into Eq. (37),

$$
h_{T}=n_{A}-\frac{u_{A} \Delta t}{x_{B}-x_{A}}\left(h_{B}-n_{A}\right) .
$$

Similarly,

$$
p_{T}=p_{A}-\frac{u_{A} \Delta t}{x_{B}-x_{A}}\left(p_{B}-p_{A}\right) .
$$

Neglecting heat addition and friction, the energy equation, Eq. (19), becomes

$$
\mathrm{dh}-\frac{1}{\rho} d p=0 .
$$

Equation (40) can be approximated with the finite difference expression

$$
n_{P}-n_{T}-\frac{1}{p_{A}}\left(p_{P}-p_{T}\right)=0 \text {. }
$$

Substituting Eqs. (38) and (39) into Eq. (41) and rearranging,

$$
h_{P}=h_{A}-\frac{u_{A} \Delta t}{x_{B}-x_{A}}\left(h_{B}-n_{A}\right)+\frac{1}{\rho_{A}}\left(p_{P}-p_{A}+\frac{u_{A} \Delta t}{x_{B}-x_{A}}\right) .
$$

IV. MODEL VERIFICATION

The preceding finite difference equations were coded into a subroutine called TEE and incorporated into the COMPATE-Mod 2 code. A statement was added to subroutine VOLCON to call subroutine EQUILB, and a call to subroutine TEE 
was made from subroutine ENTRAN when the boundary conditions at tie branch point were to be calculated. A listing of subroutine TEE can be found in the Appendix.

Verification of the branch flow model was achieved by comparison of calculated results at steady state with air flow experimerits through a tee wich three flow junctions of equal diameter $(31.75 \mathrm{~mm}) .^{5}$ The tee-flow model geometry and initial conditions are shown schematically in Fig. 4. Air properties were input at $294.4 \mathrm{~K}$ and atmospheric pressure. Under the conditions shown in Fig. 4, the air flowed from Volume 1 through Volume 4 and into Volumes 2 and 3 at equal rates and the flow was incompressible. The COMPARE code flips the coordinates in Junction 1 when calculating the boundary condition at Volume 4 so that the branch point becrmes the first node, and Family II characteristics equations can be used as developed in Sec. II:.A.

The results of the model caiculations are shown in Fig. 5. The loss coefficients defined in Eq. (27) were varied until agreement was achieved with experimental data. In this limited investigation, all three loss coefficients were kept equal, but, in general, they would not have to be equal. Good agreement was obtained with experimental data when loss coefficients of 0.3 were used. With the loss coefficients maintained at 0.3 , the pressure in Volume 1 was both

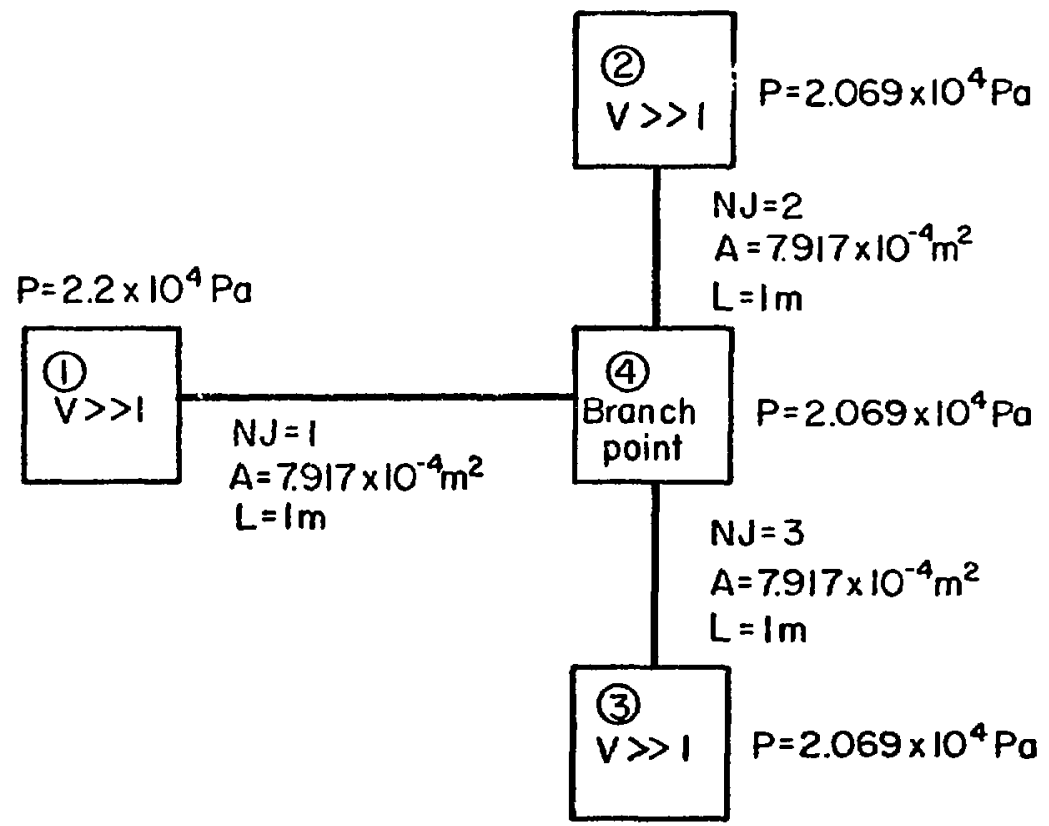

Fig. 4. Volume and junction geometry and initial conditions used in tee-flow calculations ( $N J$ is junction number, circled numbers designate volumes). 

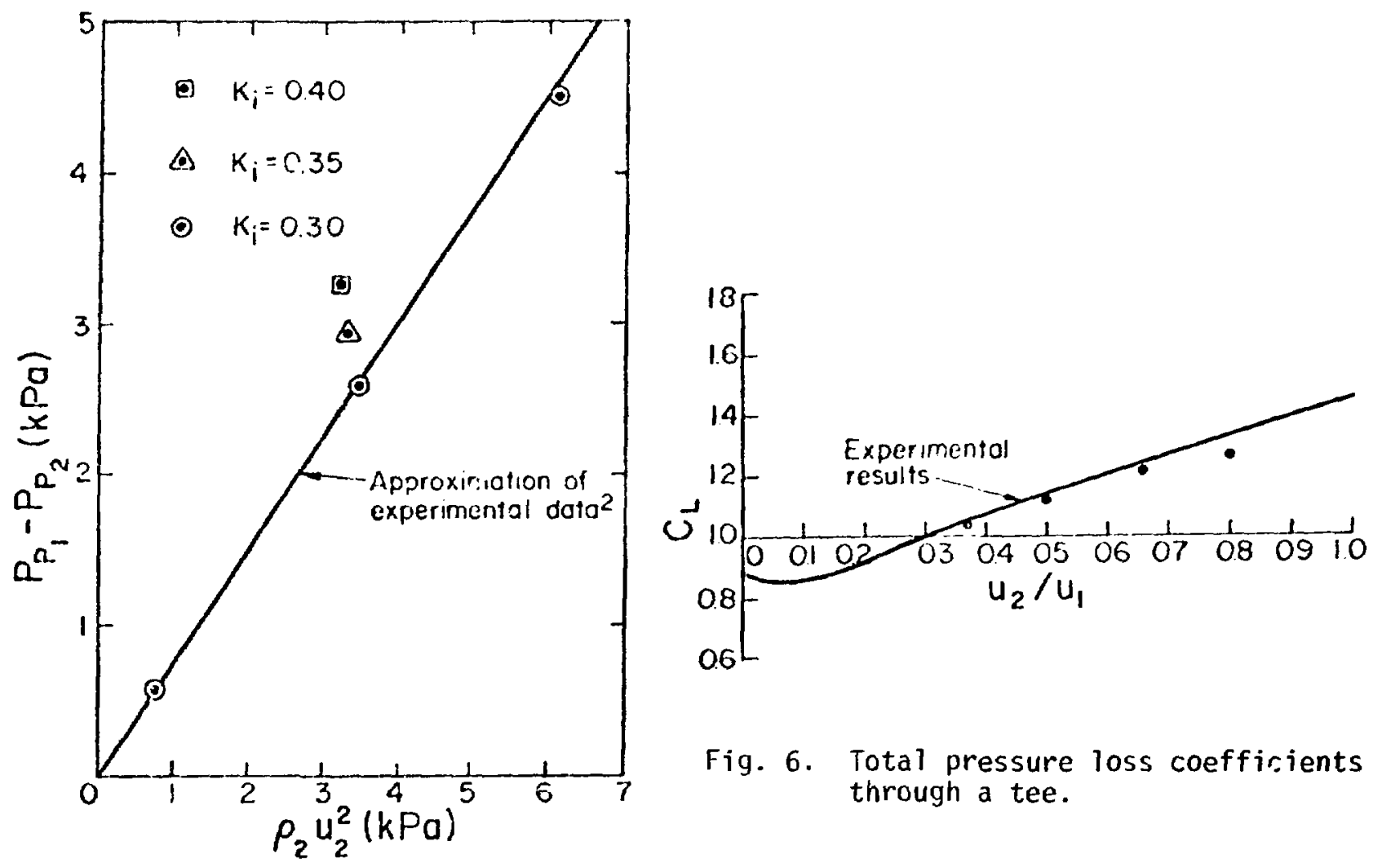

Fig. 6. Total pressure loss coeffirients through a tee.

Fig. 5. Static pressure drop through a tee.

increased and decreased to obtain different flow rates in the junctions. These points are ilso shown in Fig. 5 and agree well with published experimental data.

For purposes of additional comparison with experimental data, a nondimensional loss coefficient was calculated using total pressures.

$$
c_{L}=\frac{p_{P_{1}}+\frac{1}{2} \rho_{A_{1}} u_{P_{1}}^{2}-\left(p_{P_{2}}+\frac{1}{2} \rho_{A_{2}} u_{A_{2}}^{2}\right)}{\frac{1}{2} \rho_{A_{1}} u_{P_{1}}^{2}} .
$$

Figure 6 shows the effect of variation of the Junction 3 flow area on the nondimensional total pressure drop defined by Eq. (43). The loss coefficients were maintained at 0.3 in all four cases. The results are in good agreement with the experimental data. 


\section{CONCLUSIONS}

The characteristic equations and finite difference approximations developed in Sec5. II and 111 were used to satisfactority model the flow of air through a branching tee. The results are in good agreement with published experimental data. The formulation can be extended to model flow through a branch point with four or more junctions by simple extension of the equations developed herein.

It should be noted that considerable scatter in experimental data gathered by various authors was nuted by Benson and Woollatt. ${ }^{5}$ The construction of the flow branches, i.e., sharp or rounded edges, had a great effect on the observed pressure drops. These variations in tee construction can be accounted for by changing the pressure loss coefficient in Eq. (27).

NOMENCLATURE

a Polytropic exponent

A Area

C Sonic velocity

$C_{L}$ Pressure loss coefficient

$D$ Equivalent diameter

$f$ Friction factor

$F \quad$ Friction term in momentum equation

h Specific enthalpy

$k$ Ratio of specific heats

$K \quad$ Static pressure loss coefficient

p Pressure

P Branch point, branch central pressure

Q Heat addition term in energy equation, Eq. (4)

t Time

$\Delta t \quad$ Time increment in finite difference formulation

$u$ Velocity in direction of flow

$x$ Coordinate in direction of flow

$Z_{s} \quad$ Nondimensional quantity defined by Eq. (21)

$\alpha \quad$ Angle between gravity vector and flow direction

B Quantity defined by Eq. (30)

$\gamma \quad$ Quantity defined by Eqs. (13) and (31) 
$\lambda \quad$ Quantity defined by Eq. (13).

$\rho \quad$ Fluid density

Subscripts:

A,B, Points defined by Fig. 2

E Exit condition

I Inlet condition

$i$ Junction number

\section{REFERENCES}

1. A. H. Shapiro, The Dynamics and Thermodynamics of Compressible Fluid Flow, Vol. II (Ronald Press Co., New York, 1954).

2. R. G. Gido, C. I. Grimes, R. G. Lawton, and J. A. Kudrick, "ComparE: A Computer Program for the Transient Calculation of a System of Volumes Connected by Flowing Vents," Los Alamos Scientific Laboratory report LA-NUREG-6488-MS (September 1976).

3. R. G. Gido, J. S. Gilbert, R. G. Lawton, and W. L. Jensen, "CoMPARE-Mod 1: A Code for the Transient Analysis of Volumes with Heat Sinks, Flowing Vents, and Doors," Los Alamos Scientific Laboratory report (to be publ ished).

4. V. L. Streeter and E. B. Wylie, Hydraul ic Transients (McGraw-Hi11, 1967).

5. R. S. Benson and D. Woollatt, "Compressible Flow Loss Coefficients at Bends and T-Junctions," The Engineer (January 28, 1966).

\section{APPENDIX}

\section{SUBROUTINE TEE}

The following is a listing of subroutine TEE. The subroutine is a FORTRAN formulation of the finite difference equations developed in Sec. III and was appended to the COMPARE-Mod 2 code for calculation of flow branching. 


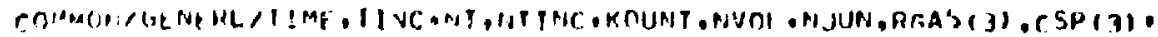

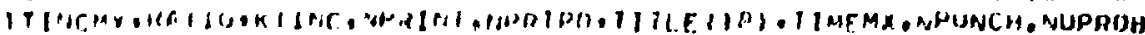

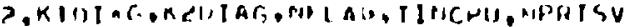

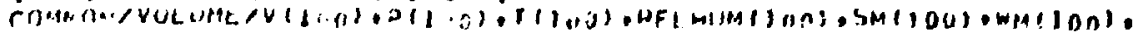

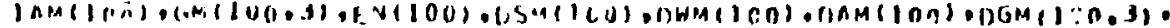
PnE $M\left(I^{\circ}(\cdot)\right.$

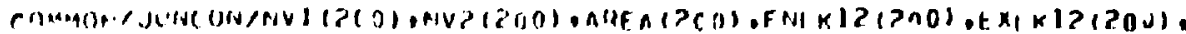

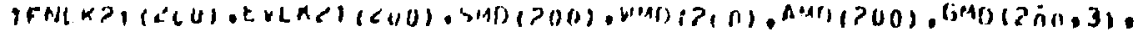

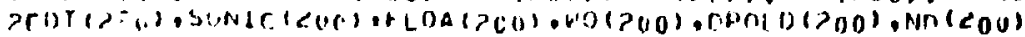

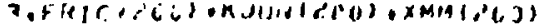

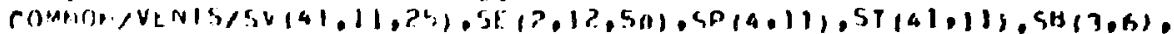

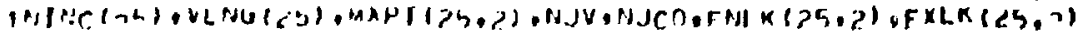

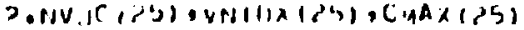

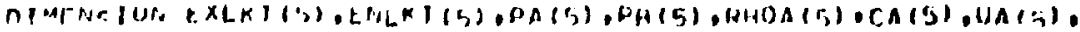

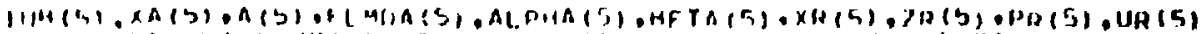

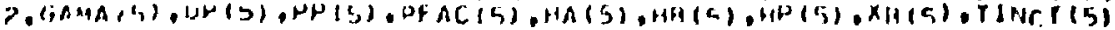

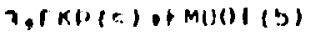

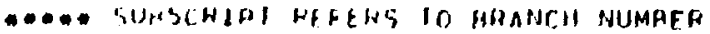

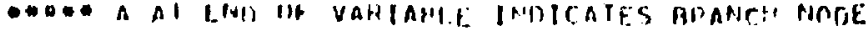

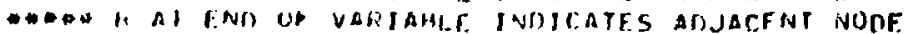

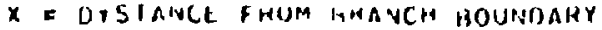

D STAIIC RHESSUHE

II = ViLUC, IIY

$r=\sin t_{4} \downarrow C$ VELOC $\downarrow$ IY

BHC $=$ H(1111) UENSITY

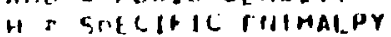

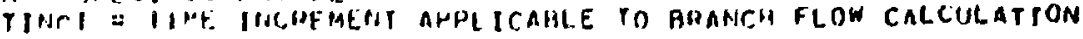

FKP a PKE SPIJMF LUSS COEFF ICIENT

PrAN = MUACCN CEMIHAL PUESSUAF

IIf I 1 I I

NLEREA

(i) $10190000 \mathrm{~J}, 0001 . \mathrm{NJ}$

c

c

(9) $\left.x p_{n}\right)=r v(1,4,1)$

CA(I) $F \times(-K T, 1)=t(1 . K) 2(1)$

CMCBN $\Gamma: 1 . K T, \vec{B})=E N L K I L(2)$

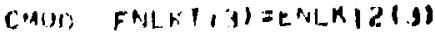

FKP 1 I) $=$ XLKILII)

FKP, $P)=F N(. K)<(2)$

FKP, $31:$ ENLKI:(3)

rin if $1=1$, NL.tio

पAII)

$x+(1)=3 v(<+1 \cdot 1)$

DA $(1)=5 \vee(1,6,1)$

PHs (1):SV (2,6, I)

in $(1)+5 \vee(1 \cdot 3 \cdot 1)$

$11+1)=5 \vee(2,3 \cdot 1)$

$r A(1)-3 V(1.4,1)$

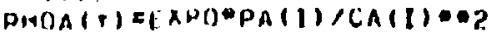

$H A(1)=S \vee(1 \cdot 1,1)-0.5 * 5 \vee(1,3,1) * 42$

$H^{H}(1)=5 \vee(\alpha, I, 1)-0.5 * 5 \vee(2,3,1) * 2$

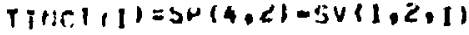

c

as CONYINUt

C

- OH. CALCHLATIUNS

$F X P \cap F=E X P U /\left(t \times F^{\prime} U=1.\right)$

กO $\ln \bar{A} 1=1$ OHLEG

C

TOG * PUL Y IPUPIC EXPONEN

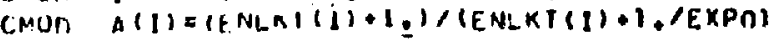

comon rin to $I<C$

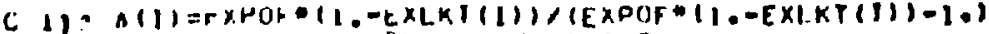

C

- ano pléliminarer calculations
GFNF MI, 2 GFIFPL 3 GFMFHL VOL UME: $?$ VOLUME 3 VOI INAF

Jurson 2 jusiron 1 JINCON 4 JuRJCON 4 VENTS $Z$ VEATS 3 VFIIT 4 


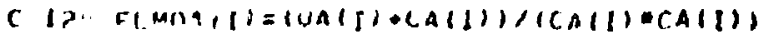

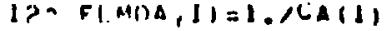

CMUN ALPHA I I $=(1.0$ (1.)

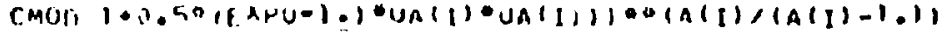

al

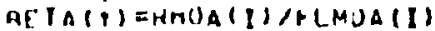

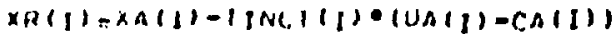

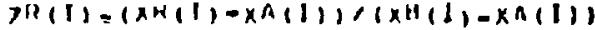

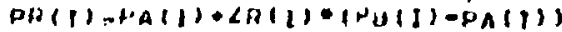

IH (I) $=U A(I) \cdot C A(I) \cdot(U)(I)-1] A(I))$

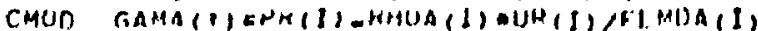

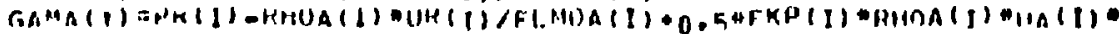

ints (UnA)

In corditilit

nO $20 \hat{A} \quad 1=1$.NLEG

PFAC (I) DAHEA(I) WHOHA (I) /HLTAII)

ina rONI InUt

$210 n=0$.

$n=0$.

ON $75 \div \quad 1=1$, NLEE

$O=D \cdot H_{F} A C(1) \cup($ AMA $(I)$

OAH.PFAC(1) ALHHA II)

zas rONIINUE

PCAPEOAH

C

* ** veluci!r ano rhessiJaf

$F M \cap F F=10$.

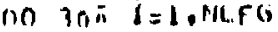

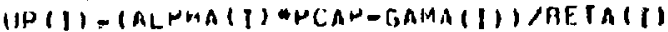

PP( )

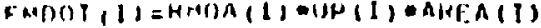

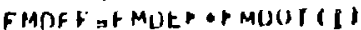

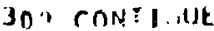

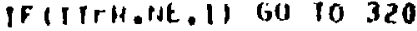

C

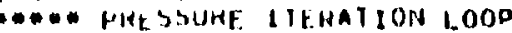

ITHAD

nO $11^{*}$ IEI.NLFG;

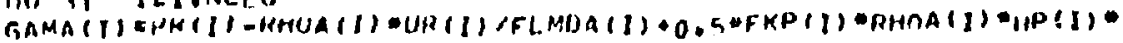

lais 11001$)$ !

II ront InUte

C

go 10 ? 10

$\operatorname{sic} 0=0$.

-*:- ENIHALFY

$A=0$.

no in: $1=1$ INLEG

If $(1), 1) \cdot(1,0.0$ (j) In 400$)$

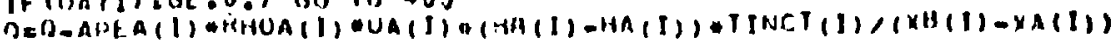

$Q=R+A \cap E A(i) \cdot$ HHOA $(I)$

$4 \mathrm{C}^{\circ}$ CONI INUE

$H T=0$.

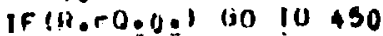

$H T=(3 / n$

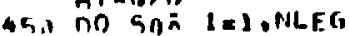

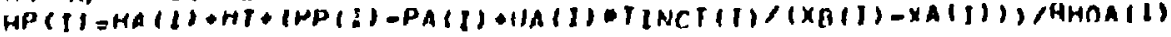

SO CONTIRIJE

WRITE, 10,10101 UP,PP. HP,FMDEF

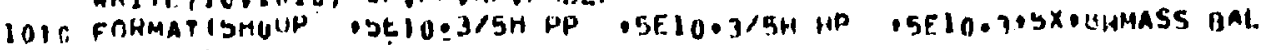
I, EIOP.T)

$\stackrel{c}{c}$

$\cos 5 P(4,7)=0 \%(N J)$

$\operatorname{SP}(4, R)=R(N)$

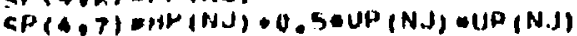

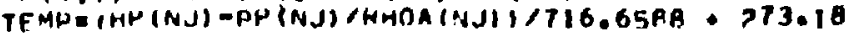

SP( $4 . A)=S U H)\left(2.80,66^{\circ} E \times P O *\right.$ TEMP)

GP $(4, E)=E \times N O$

o(4) IIDCAP

IF (NI.L!・HOA) HE!UAN 


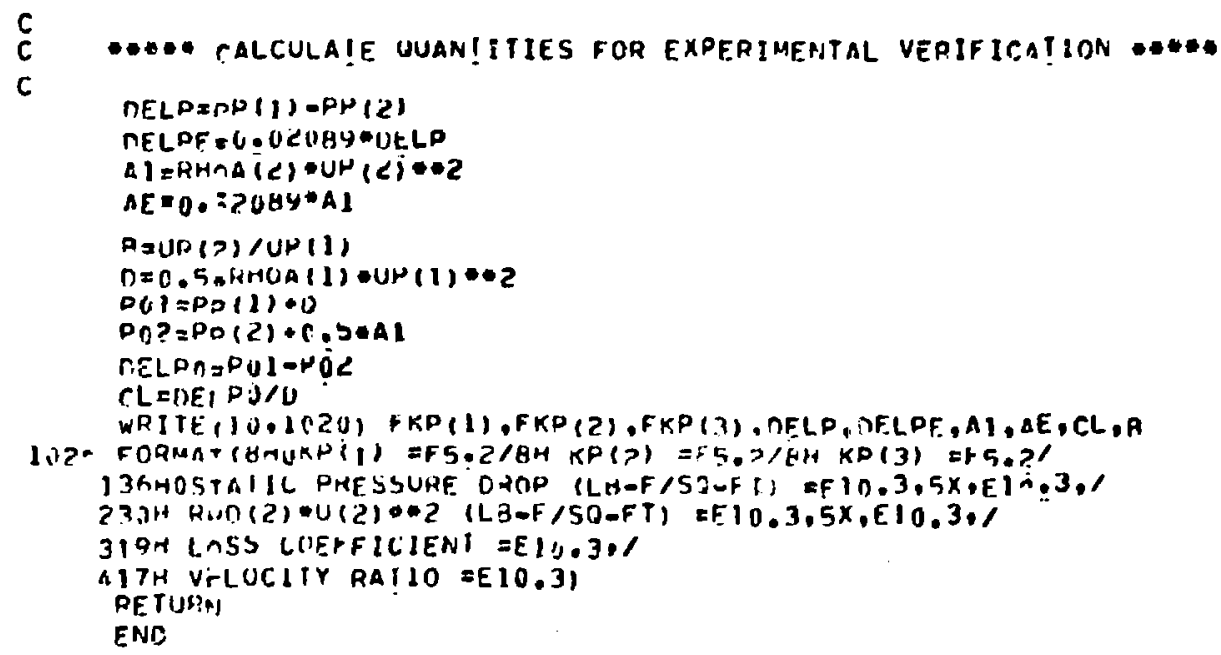

